\title{
LINEAR DIFFERENCE EQUATIONS AND PERIODIC SEQUENCES OVER FINITE FIELDS
}

\author{
Dang Vu Giang \\ Hanoi Institute of Mathematics \\ 18 Hoang Quoc Viet, 10307 Hanoi, Vietnam \\ e-mail: 〈dangvugiang@yahoo.com〉
}

May 21, 2018

\begin{abstract}
First, we study the linear equations in general. A common period is found for every solution of a linear difference equation over a finite field. It will help to estimate the $p$-adic modulus of polynomial roots. Second, we focus our attention in periodic sequences over finite fields and Hamilton cycles in de Bruijn directed graph.
\end{abstract}

2000 AMS Subject Classification: 12E20, 12Y05

KEY WoRDS: Jordan multiplicative decomposition, characteristic equations, Lucas' congruence, minimal polynomial, trace representation, maxsequences 


\section{LINEAR DIFFERENCE EQUATIONS OVER P-ADIC FIELD AND CONVERGENCE}

Recall that the $p$-field $\mathbb{C}_{p}$ of $p$-adic numbers is algebraically closed (see [13]). Hence, every invertible $p$-adic matrix can be written in the form $A=S U=U S$ where $S$ is simple matrix and $U$ is unipotent matrix (see [5]). This form is called the Jordan multiplicative decomposition of an invertible matrix $A$. Theorems of Lie and Engel are true for Lie algebras over $p$-adic fields. Also Killing form and semisimple Lie algebra can be defined over $p$-adic fields. We are interested in the discrete dynamics of $p$-adic matrices and Haar measure of $p$-adic integers. Unlike complex matrices, $p$-adic matrices have only discrete dynamical version. (We have no sense of real and image parts of a $p$-adic number.) Consider the difference equation $\mathbf{v}_{n+1}=A \mathbf{v}_{n}$ for $n=0,1,2, \cdots$, where $\mathbf{v}_{0}$ is a given vector in a $k$-dimensional vector space over $\mathbb{C}_{p}$. Without loss of generality we assume that $A$ is an invertible matrix. We can write down $A=S U=U S$ where $S$ is a simple matrix and $U$ is a unipotent matrix (see [5]). Let $\mathbf{e}_{1}, \mathbf{e}_{2}, \cdots, \mathbf{e}_{k}$ be (linearly independent) eigenvectors of $S$. Then there are eigenvalues $\lambda_{1}, \lambda_{2}, \cdots, \lambda_{k}$ (maybe identical) of $A$ such that $S \mathbf{e}_{1}=\lambda_{1} \mathbf{e}_{1}, S \mathbf{e}_{2}=\lambda_{2} \mathbf{e}_{2}, \cdots, S \mathbf{e}_{k}=\lambda_{k} \mathbf{e}_{k}$. Decompose $\mathbf{v}_{0}=\alpha_{1} \mathbf{e}_{1}+\alpha_{2} \mathbf{e}_{2}+\cdots+\alpha_{k} \mathbf{e}_{k}$ where $\alpha_{1}, \alpha_{2}, \cdots, \alpha_{k} \in \mathbb{C}_{p}$. We have

$$
\begin{aligned}
\mathbf{v}_{n} & =\alpha_{1} U^{n} S^{n} \mathbf{e}_{1}+\alpha_{2} U^{n} S^{n} \mathbf{e}_{2}+\cdots+\alpha_{k} U^{n} S^{n} \mathbf{e}_{k} \\
& =\alpha_{1} \lambda_{1}^{n} U^{n} \mathbf{e}_{1}+\alpha_{2} \lambda_{2}^{n} U^{n} \mathbf{e}_{2}+\cdots+\alpha_{k} \lambda_{k}^{n} U^{n} \mathbf{e}_{k},
\end{aligned}
$$

which tends to zero vector as $n \rightarrow \infty$ if $p$-adic absolute values of $\lambda_{1}, \lambda_{2}, \cdots, \lambda_{k}$ are less than 1 . Indeed, $U=I+N$ ( $N$ is nilpotent, $I$ is identity matrix) so

$$
\lambda^{n} U^{n} \mathbf{e}=\lambda^{n}(I+N)^{n} \mathbf{e}=\sum_{\ell=0}^{k-1} \frac{n(n-1) \cdots(n-\ell+1)}{\ell !} \cdot \lambda^{n} N^{\ell} \mathbf{e}
$$

which is certainly tends to 0 as $n \rightarrow \infty$ if $|\lambda|_{p}<1$, because

$$
\frac{n(n-1) \cdots(n-\ell+1)}{\ell !} \cdot|\lambda|_{p}^{n} \rightarrow 0
$$

as for $n \rightarrow \infty$ for any fixed $\ell$. We have 
Theorem 1. If the p-adic absolute value of every eigenvalue of a $p$-adic matrix $A$ is less than 1 then every solution of $\mathbf{v}_{n+1}=A \mathbf{v}_{n}$ converges to $\mathbf{0}$ as $n \rightarrow \infty$.

Remark. If the $p$-adic matrix $A$ is not invertible we decompose the $k-$ dimensional space (over $\mathbb{C}_{p}$ )

$$
V=V_{0} \oplus \operatorname{Ker}(A)
$$

and set $A_{0}=\left.A\right|_{V_{0}}$. Then $A_{0}$ is invertible and we process with $A_{0}$ and exactly as with $A$ and $V$.

\section{THE PERIOD OF LINEAR DIFFERENCE EQUA- TIONS OVER FINITE FIELDS}

Consider the difference equation $\mathbf{v}_{n+1}=A \mathbf{v}_{n}$ for $n=0,1,2, \cdots$, where $\mathbf{v}_{0}$ is a given vector in a $k$-dimensional vector space over $\mathbb{F}_{q}(q$ is a power of a prime $p)$. First we assume that the matrix $A$ is invertible. Then $A=S U=U S$ where $S$ is simple matrix and $U$ is unipotent matrix over an extension of the field $\mathbb{F}_{q}$ (see Goodman et al). We have

$$
\begin{aligned}
\mathbf{v}_{n} & =\alpha_{1} U^{n} S^{n} \mathbf{e}_{1}+\alpha_{2} U^{n} S^{n} \mathbf{e}_{2}+\cdots+\alpha_{k} U^{n} S^{n} \mathbf{e}_{k} \\
& =\alpha_{1} \lambda_{1}^{n} U^{n} \mathbf{e}_{1}+\alpha_{2} \lambda_{2}^{n} U^{n} \mathbf{e}_{2}+\cdots+\alpha_{k} \lambda_{k}^{n} U^{n} \mathbf{e}_{k},
\end{aligned}
$$

where $\mathbf{e}_{1}, \mathbf{e}_{2}, \cdots, \mathbf{e}_{k}$ are (linearly independent) eigenvectors of $S$ and $\lambda_{1}, \lambda_{2}$, $\cdots, \lambda_{k}$ are eigenvalues of $A$ (in a finite extension of $\mathbb{F}_{q}$ ). Moreover, $U=N+I$ ( $N$ is nilpotent, $I$ is identity matrix) so

$$
\lambda^{n} U^{n} \mathbf{e}=\lambda^{n}(I+N)^{n} \mathbf{e}=\sum_{\ell=0}^{k-1} \frac{n(n-1) \cdots(n-\ell+1)}{\ell !} \cdot \lambda^{n} N^{\ell} \mathbf{e} .
$$

A lot is known about the periods of vector sequences over $\mathbb{F}_{q}$ defined by a recurrence $\mathbf{v}_{n+1}=A \mathbf{v}_{n}$ with a matrix $A$, see for example [4] or Chapter 10.1 of [11]. However, the explicit formula of the period is found for irreducible characteristic polynomials only. Here we give the explicit formula of the period of $\mathbf{v}_{n}$ not only in that case but for arbitrary characteristic polynomial. 
Our method is based on Lucas' congruence and writing the dimension $k$ in $p$-adic base. Indeed, the period of $\left(\begin{array}{l}n \\ \ell\end{array}\right) \bmod p$ is $p$ if $\ell<p$. Clearly, $\lambda_{j}$ is root of the characteristic equation $\operatorname{det}(A-\lambda I)=0$ (in an extension of $\left.\mathbb{F}_{q}\right)$. Consider the characteristic equation $\operatorname{det}(A-\lambda I)=0$ in an extension of $\mathbb{F}_{q}$ and let $\left\{\varphi_{j}\right\}_{j=1}^{N} \subseteq \mathbb{F}_{q}[x]$ are irreducible factors (of the characteristic polynomial of the matrix $A$ ). If $k_{j}$ is degree of $\varphi_{j}$ then $\varphi_{j}$ is a divisor of $x^{q^{k_{j}}}-x$ (see [10] for more details). Let $Q$ be the smallest multiplier of $q^{k_{j}}-1$ then every root $\lambda$ of the characteristic equation $\operatorname{det}(A-\lambda I)=0$ satisfies $\lambda^{Q}=1$ so the period of $\mathbf{v}_{n+1}=A \mathbf{v}_{n}$ is $Q$ if there is no duplicated root $(U=I$ in this case). If there is a duplicated root, we should make an intention about the periodicity of sequences $\left(\begin{array}{l}n \\ \ell\end{array}\right)$ of $n$ in $\mathbb{F}_{q}$. By Lucas' congruence

$$
\left(\begin{array}{c}
n \\
j
\end{array}\right) \equiv\left(\begin{array}{c}
n_{0} \\
j_{0}
\end{array}\right) \cdots\left(\begin{array}{c}
n_{r} \\
j_{r}
\end{array}\right) \quad \bmod p
$$

if $n=n_{0}+\cdots+n_{r} p^{r}+\cdots$ and $j=j_{0}+\cdots+j_{r} p^{r}$ with $0 \leq n_{i}, j_{i}<p$. Hence, if we write $k-1=\kappa_{0}+\cdots+\kappa_{r} p^{r}$ then the period of sequences $\left(\begin{array}{l}n \\ \ell\end{array}\right)$ $(0 \leq \ell \leq k-1)$ is $p^{r+1}$.

Theorem 2. Assume that $A$ is $k \times k$ invertible matrix over $\mathbb{F}_{q}$. Let $k_{1}<k_{2}<$ $\cdots<k_{N}$ be degrees of irreducible factors of the characteristic polynomial of $A$ and let $Q=Q(A)$ be the smallest multiplier of $q^{k_{j}}-1$. Then every solution of the linear system $\mathbf{v}_{n+1}=A \mathbf{v}_{n}$ over $\mathbb{F}_{q}$ is $Q$-periodic if the multiplicity of every irreducible factor is 1 . Specially if the characteristic polynomial of $A$ is irreducible then $q^{k}-1$ is a common period of every solution of the linear system $\mathbf{v}_{n+1}=A \mathbf{v}_{n}$ over $\mathbb{F}_{q}$. If the multiplicity of a factor is larger than 1 then every solution of $\mathbf{v}_{n+1}=A \mathbf{v}_{n}$ is $Q p^{r+1}$-periodic, where $p$ is the characteristics of $\mathbb{F}_{q}$ and the exponent $r$ is determined by writing $k-1=$ $\kappa_{0}+\cdots+\kappa_{r} p^{r}$ with $0 \leq \kappa_{i}<p$ and $0<\kappa_{r}<p$.

Remark. If the matrix $A$ is not invertible we decompose the $k$ - dimensional space (over $\left.\mathbb{F}_{q}\right) V=V_{0} \oplus \operatorname{Ker}(A)$ and set $A_{0}=\left.A\right|_{V_{0}}$. Then $A_{0}$ is invertible and we process with $A_{0}$ exactly as with $A$. The system $\mathbf{v}_{n+1}=A \mathbf{v}_{n}$ is $\rho$-like periodic, that is there is a period $T \in\left\{Q\left(A_{0}\right), p Q\left(A_{0}\right)\right\}$ such that 
$\mathbf{v}_{n+T}=\mathbf{v}_{n}$ for all but finite $n$. Specially, if $A$ is nilpotent, $\mathbf{v}_{n}=\mathbf{0}$ for $n=k, k+1, \cdots$ and the period $T=1$.

Example 1. Let $a_{n}=n$ for $n=0,1, \cdots, p-1$ and $a_{n}=a_{n-1}+a_{n-p}$ for $n=p, p+1, \cdots$. In the finite field $\mathbb{F}_{p}$ we have $a_{n}=a_{n+p^{2}-1}$. To see this, solve the equation $\lambda^{p}=\lambda^{p-1}+1$ in an extension of $\mathbb{F}_{p}$. We have $(\lambda-1)^{p}=$ $\lambda^{p-1}$ and consequently, $(\lambda-1)^{p+1}=\lambda^{p-1}(\lambda-1)=1$. Therefore, $\lambda^{p^{2}-1}=$ $(\lambda-1)^{p(p+1)}=1$. Hence $a_{n}=a_{n-1}+a_{n-p}$ is periodic in $\mathbb{F}_{p}$ with period $p^{2}-1$. This sequence is not convergent in the $p$-adic field, since $\left|a_{n+\ell\left(p^{2}-1\right)}\right|_{p}=1$ for all $\ell \in \mathbb{N}$ and $n=1, \cdots, p-1$ so $\left|a_{n+\ell\left(p^{2}-1\right)+p}-a_{n+\ell\left(p^{2}-1\right)+p-1}\right|_{p}=$ $\left|a_{n+\ell\left(p^{2}-1\right)}\right|_{p}=1$ which does not converge to 0. Hence, $a_{n}=a_{n-1}+a_{n-p}$ is not convergent in the $p$-adic field and at least one root of $\lambda^{p}=\lambda^{p-1}+1$ has $p$-adic absolute value $\geq 1$.

Example 2. Consider the equation $x_{n}=x_{n-4}+x_{n-5}$ over $\mathbb{F}_{2}$. The characteristic polynomial is $\lambda^{5}+\lambda+1=\left(\lambda^{2}+\lambda+1\right)\left(\lambda^{3}+\lambda^{2}+1\right)$. The smallest multiplier of $2^{2}-1=3$ and $2^{3}-1=7$ is 21 . Hence a period of this equation is 21 . This example is given by [1].

Example 3. Consider $x_{n}=x_{n-3}+x_{n-5}$ over $\mathbb{F}_{2}$. The characteristic polynomial is $\lambda^{5}+\lambda^{2}+1$ irreducible over $\mathbb{F}_{2}$. Hence the minimal period of any non-zero solution of this equation is $2^{5}-1=31$. This example is given by 1 .

Example 4. Consider the equation $x_{n}+x_{n+1}+x_{n+2}+x_{n+4}=0$ over $\mathbb{F}_{2}$. The characteristic polynomial is $\lambda^{4}+\lambda^{2}+\lambda+1=(\lambda+1)\left(\lambda^{3}+\lambda^{2}+1\right)$. The smallest multiplier of $2^{1}-1=1$ and $2^{3}-1=7$ is 7 . Hence the minimal period of any non-zero solution of this equation is 7 . This example is given by [1].

Example 5. Consider the Fibonacci sequence $x_{n}=x_{n-1}+x_{n-2}$ over $\mathbb{F}_{q}$. The characteristic polynomial is $\lambda^{2}-\lambda-1$. If this polynomial is irreducible over $\mathbb{F}_{q}$ then the Fibonacci sequence is $\left(q^{2}-1\right)$-periodic. Otherwise, it is $(q-1)-$ or $(q-1) p$-periodic. For example, $\lambda^{2}-\lambda-1$ is irreducible over $\mathbb{F}_{2}$ and $\mathbb{F}_{3}$ but $\lambda^{2}-\lambda-1=(\lambda-3)^{2}$ over $\mathbb{F}_{5}$. Hence, the Fibonacci sequence is 3-periodic over $\mathbb{F}_{2}, 8$-periodic over $\mathbb{F}_{3}$ and 20 -periodic over $\mathbb{F}_{5}$. In $\mathbb{F}_{4}$, $\lambda^{2}-\lambda-1$ has two distinct roots so the Fibonacci sequence is 3 -periodic over $\mathbb{F}_{4}$. This example is solved in part by [8]. 


\section{MINIMAL POLYNOMIAL AND MINIMAL PERIOD OF PERIODIC SEQUENCES}

Let $S=\left\{s_{0}, s_{1}, \cdots\right\}$ be an arbitrary sequence over $\mathbb{F}_{q}$. Let $\mathbb{E}$ denote the shift operator on the linear space of sequences. This means that $\mathbb{E}(S)$ is the sequence whose $i$ th term is $s_{i+1}$. Moreover, $\mathbb{E}^{k}(S)$ is denoted the sequence whose $i$ th term is $s_{i+k}$. Two sequences $S=\left\{s_{0}, s_{1}, \cdots\right\}$ and $T=\left\{t_{0}, t_{1}, \cdots\right\}$ are equivalent if $\mathbb{E}^{k}(S)=T$ for some integer $k$. Suppose that

$$
s_{i+n}+c_{n-1} s_{i+n-1}+\cdots+c_{0} s_{i}=0
$$

for some elements $c_{0}, c_{1}, \cdots, c_{n-1} \in \mathbb{F}_{q}$ and for all $i=0,1, \cdots$. Then the polynomial $x^{n}+c_{n-1} x^{n-1}+\cdots+c_{0}$ is called a characteristic polynomial of $S=\left\{s_{0}, s_{1}, \cdots\right\}$. For a periodic sequence $S=\left\{s_{0}, s_{1}, \cdots\right\}$ with a period $N$ we have $s_{i+N}-s_{i}=0$, so $x^{N}-1$ is a characteristic polynomial of $S=\left\{s_{0}, s_{1}, \cdots\right\}$. A characteristic polynomial of minimal degree is called the minimal polynomial of $S=\left\{s_{0}, s_{1}, \cdots\right\}$. Any periodic non-zero sequence has one and only one minimal polynomial. The degree of the minimal polynomial is called the complexity of the periodic sequence and denoted by $c(S)$. Clearly, $c(S) \leq$ minimal period of $S=\left\{s_{0}, s_{1}, \cdots\right\}$. Moreover, the minimal polynomial divides any characteristic polynomial of $S=\left\{s_{0}, s_{1}, \cdots\right\}$. Hence, if a characteristic polynomial of a periodic sequence is irreducible then it is itself the minimal polynomial of that sequence. If $N$ is a period of $S=\left\{s_{0}, s_{1}, \cdots\right\}$ then the minimal polynomial of $S=\left\{s_{0}, s_{1}, \cdots\right\}$ is

$$
\frac{x^{N}-1}{\operatorname{gcd}\left(x^{N}-1, s_{0}+s_{1} x+\cdots+s_{N-1} x^{N-1}\right)} .
$$

Therefore,

$$
c(S)=N-\operatorname{deg} \operatorname{gcd}\left(x^{N}-1, s_{0}+s_{1} x+\cdots+s_{N-1} x^{N-1}\right) .
$$

For example, the linear complexity of the Fibonacci sequence $\{1,1,2,3, \cdots\}$ over $\mathbb{F}_{5}$ is 2 and its minimal period is 20 .

Theorem 3. The minimal period of a non-zero sequence $S=\left\{s_{0}, s_{1}, \cdots\right\}$ is a power of $p$ iff the minimal polynomial of $S=\left\{s_{0}, s_{1}, \cdots\right\}$ is a power of $(x-1)$. Therefore, if a characteristic polynomial of $S=\left\{s_{0}, s_{1}, \cdots\right\}$ is of 
the form $(x-1)^{k}$ then the minimal period of $S=\left\{s_{0}, s_{1}, \cdots\right\}$ is $p^{r+1}$ with $p^{r}<c(S) \leq p^{r+1}$, unless $c(S)=1$, in which case $S=\{s, s, \cdots\}$ and the minimal period of $S$ is 1 . Recall that $p$ is the characteristics of the finite field $\mathbb{F}_{q}$.

Proof: If $p^{r+1}$ is the minimal period of $S=\left\{s_{0}, s_{1}, \cdots\right\}$ then

$$
x^{p^{r+1}}-1=(x-1)^{p^{r+1}}
$$

is a characteristic polynomial of $S=\left\{s_{0}, s_{1}, \cdots\right\}$. Therefore, $(x-1)^{c(S)}$ is the minimal polynomial of $S=\left\{s_{0}, s_{1}, \cdots\right\}$. Conversely, if the minimal polynomial of $S=\left\{s_{0}, s_{1}, \cdots\right\}$ is $(x-1)^{c(S)}$ then the minimal period of $S=\left\{s_{0}, s_{1}, \cdots\right\}$ is a power of $p$. Indeed, if $c(S)=1$ then $S=\{s, s, \cdots\}$ and the minimal period is 1 . For $c(S)>1$ choose an integer $r$ such that $p^{r}<c(S) \leq p^{r+1}$ (this integer $r$ is unique). Then $(x-1)^{p^{r+1}}=x^{p^{r+1}}-1$ is a characteristic polynomial of $S=\left\{s_{0}, s_{1}, \cdots\right\}$ so $p^{r+1}$ is a period of $S=\left\{s_{0}, s_{1}, \cdots\right\}$. Moreover, $p^{r+1}$ is the minimal period of $S=\left\{s_{0}, s_{1}, \cdots\right\}$ because $c(S)>p^{r}$. The proof is complete.

Remark. These results can be found in [3] with more details. Recall that the weight of a periodic sequence $S=\left\{s_{0}, s_{1}, \cdots\right\}$ with minimal period $t$ is the sum

$$
s_{1}+s_{2}+\cdots+s_{t}
$$

Proposition. If the minimal period of $S=\left\{s_{0}, s_{1}, \cdots\right\}$ is $p^{r+1}$ then $c(S)=$ $p^{r+1}$ if and only if the weight of $S=\left\{s_{0}, s_{1}, \cdots\right\}$ is non-zero.

Proof: If

$$
s_{1}+s_{2}+\cdots+s_{p^{r+1}}=0
$$

then

$$
s_{n+1}+s_{n+2}+\cdots+s_{n+p^{r+1}}=0
$$

for every $n=0,1, \cdots$, so $x^{p^{r+1}-1}+x^{p^{r+1}-2}+\cdots+1$ is a characteristic polynomial and consequently, $c(S)<p^{r+1}$. If

$$
s_{1}+s_{2}+\cdots+s_{p^{r+1}} \neq 0
$$

then

$$
s_{n+1}+s_{n+2}+\cdots+s_{n+p^{r+1}} \neq 0
$$


for every $n=0,1, \cdots$, so $x^{p^{r+1}-1}+x^{p^{r+1}-2}+\cdots+1=(x-1)^{p^{r+1}-1}$ is not a characteristic polynomial and consequently, $c(S)=p^{r+1}$. The proof is complete.

Remark. This proposition is proved in [12] by other way.

Proposition. Let $m_{1}, m_{2}, \cdots, m_{h}$ be minimal polynomials of periodic sequences $S_{1}, S_{2}, \cdots, S_{h}$ and assume that they are pairwise relatively prime. Then the minimal polynomial of $S_{1}+S_{2}+\cdots+S_{h}$ is $m_{1} m_{2} \cdots m_{h}$.

Proof: By complete induction, it is enough to consider the case $h=2$. Let $N$ be a common period of $S_{1}$ and $S_{2}$. Then

$$
m_{1}(x)=\frac{x^{N}-1}{\operatorname{gcd}\left(x^{N}-1, S_{1}(x)\right)} \text { and } m_{2}(x)=\frac{x^{N}-1}{\operatorname{gcd}\left(x^{N}-1, S_{2}(x)\right)} .
$$

On the other hand, we can write $x^{N}-1=f_{1} m_{1}=f_{2} m_{2}$ and $S_{1}=f_{1} g_{1}$, $S_{2}=f_{2} g_{2}$ with gcd $\left\{m_{1}, g_{1}\right\}=\operatorname{gcd}\left\{m_{2}, g_{2}\right\}=1$. But $m_{1}$ and $m_{2}$ are co-prime so $f_{1}=m_{2} \delta$ and $f_{2}=m_{1} \delta$ and we have $x^{N}-1=m_{1} m_{2} \delta$ and $S_{1}+S_{2}=$ $\left(m_{2} g_{1}+m_{1} g_{2}\right) \delta$. On the other hand the minimal polynomial of $S_{1}+S_{2}$ is

$$
\begin{aligned}
\frac{x^{N}-1}{\operatorname{gcd}\left(x^{N}-1, S_{1}(x)+S_{2}(x)\right)} & =\frac{m_{1} m_{2} \delta}{\operatorname{gcd}\left(m_{1} m_{2} \delta,\left(m_{2} g_{1}+m_{1} g_{2}\right) \delta\right)} \\
& =m_{1} m_{2} .
\end{aligned}
$$

The proof is now complete.

Definition. For a monic polynomial $f \in \mathbb{F}_{q}[x]$ we define $\mathcal{M}(f)$ to be the vector space of periodic sequences having $f$ as a characteristic polynomial. Then the dimension of $\mathcal{M}(f)$ over $\mathbb{F}_{q}$ is $\operatorname{deg}(f)$. Moreover, $\mathcal{M}(f)$ is invariant under the shift operator $\mathbb{E}$.

Proposition. Let $f_{1}, f_{2}, \cdots, f_{h} \in \mathbb{F}_{q}[x]$ be monic polynomials and pairwise relatively prime. Then $\mathcal{M}\left(f_{1} f_{2} \cdots f_{h}\right)=\mathcal{M}\left(f_{1}\right) \oplus \mathcal{M}\left(f_{2}\right) \oplus \cdots \oplus \mathcal{M}\left(f_{h}\right)$.

Proof: By complete induction, it is enough to consider the case $h=2$. If $S \in M\left(f_{1} f_{2}\right)$ then $f_{2}(\mathbb{E}) S \in \mathcal{M}\left(f_{1}\right)$ and $f_{1}(\mathbb{E}) S \in \mathcal{M}\left(f_{2}\right)$. Recall that $\mathbb{E}$ is the shift operator on the vector spaces of periodic sequences. On the other hand, since $f_{1}, f_{2}$ are relatively prime, there are $g_{1}, g_{2} \in \mathbb{F}_{q}[x]$ such that 
$f_{1} g_{1}+f_{2} g_{2}=1$. Therefore, $S=g_{1} f_{1}(\mathbb{E}) S+g_{2} f_{2}(\mathbb{E}) S \in \mathcal{M}\left(f_{1}\right) \oplus \mathcal{M}\left(f_{2}\right)$. The proof is now complete.

Proposition. Let $m=m_{1} m_{2} \cdots m_{h}$ be the minimal polynomial of a periodic sequence $S$, where $m_{1}, m_{2}, \cdots, m_{h}$ are monic polynomials and pairwise relatively prime. Then there are unique periodic sequences $S_{1}, S_{2}, \cdots, S_{h}$ with minimal polynomials $m_{1}, m_{2}, \cdots, m_{h}$, respectively, such that

$$
S_{1}+S_{2}+\cdots+S_{h}=S .
$$

Proof: By the above proposition, $S=S_{1}+S_{2}+\cdots+S_{h}$ with $S_{j} \in$ $\mathcal{M}\left(m_{j}\right)$ for $j=1,2, \cdots, h$. Let $\mu_{1}, \mu_{2}, \cdots, \mu_{h}$ be the minimal polynomials of $S_{1}, S_{2}, \cdots, S_{h}$, respectively. Then $\mu_{j} \mid m_{j}$ for $j=1,2, \cdots, h$ and the minimal polynomial of $S=S_{1}+S_{2}+\cdots+S_{h}$ is $m=m_{1} m_{2} \cdots m_{h}=\mu_{1} \mu_{2} \cdots \mu_{h}$. Therefore, $\mu_{j}=m_{j}$ for $j=1,2, \cdots, h$ and the minimal polynomials of $S_{1}, S_{2}, \cdots, S_{h}$ are $m_{1}, m_{2}, \cdots, m_{h}$, respectively. The proof is now complete.

Definition. Let $\sigma: \mathbb{F}_{q}[x] \rightarrow \mathbb{F}_{q}[x]$ be the linear mapping defined by $\sigma\left(\sum c_{j} x^{j}\right)=\sum c_{j}^{p} x^{j}$. Recall that $p$ is the characteristics of the finite field $\mathbb{F}_{q}$. Then $\sigma$ is also multiplicative map that is $\sigma(f g)=\sigma(f) \sigma(g)$. Moreover, note that the set $\left\{a \in \mathbb{F}_{q}: a^{p}=a\right\}$ is a field of $p$ elements so $\sigma(f)=f$ iff $f \in \mathbb{F}_{p}[x]$ i.e., the coefficients of $f$ are in the prime field $\mathbb{F}_{p}$. Let $k(f)$ denote the smallest integer $\ell$ such that $\sigma^{\ell}(f)=f$. Clearly, the set $\left\{a \in \mathbb{F}_{q}: a^{p^{k^{(f)}}}=a\right\}$ is a field of $p^{k(f)}$ elements so $f \in \mathbb{F}_{p^{k(f)}}[x]$. Moreover, if $q=p^{n}$ then $k(f) \mid n$.

Proposition. A polynomial $f \in \mathbb{F}_{q}[x]$ is irreducible if and only if $\sigma(f)$ is irreducible.

Proof: If $f=u v$ is reducible then $\sigma(f)=\sigma(u) \sigma(v)$ is also reducible. Conversely, if $\sigma(f)=\mu \nu$ is reducible then $f=\sigma^{\ell}(f)=\sigma^{\ell-1}(\mu \nu)=$ $\sigma^{\ell-1}(\mu) \sigma^{\ell-1}(\nu)$ is also reducible. The proof is now complete.

Proposition. If $f(x) \in \mathbb{F}_{q}[x]$ is a monic irreducible polynomial then

$$
g=f \sigma(f) \sigma^{2}(f) \cdots \sigma^{k(f)-1}(f)
$$

is in $\mathbb{F}_{p}[x]$ and irreducible over the prime field $\mathbb{F}_{p}$. Recall that $k(f)$ denotes the smallest integer $\ell$ such that $\sigma^{\ell}(f)=f$. 
Proof: Note that $\sigma(g)=\sigma(f) \sigma^{2}(f) \cdots \sigma^{k(f)}(f)=g$ so $g \in \mathbb{F}_{p}[x]$. Moreover, we can decompose $f(x)=\left(x-\alpha_{1}\right)\left(x-\alpha_{2}\right) \cdots\left(x-\alpha_{n}\right)$ where $\alpha_{j} \in \mathbb{F}_{q^{n}}$ for $j=1,2, \cdots, n$. Then $f$ is the minimal polynomial of $\alpha_{1}$. Let $\gamma$ denote the minimal polynomial of $\alpha_{1}$ over the prime field $\mathbb{F}_{p}$. Then $\gamma \mid g$ and $\gamma$ is irreducible over the prime field $\mathbb{F}_{p}$. Moreover, $f \mid \gamma$ so $\alpha_{j}, \alpha_{j}^{p}, \alpha_{j}^{p^{2}}, \cdots$ are roots of $\gamma$ for $j=1,2, \cdots, n$. Therefore, $\gamma=g$ and consequently, $g$ irreducible over the prime field $\mathbb{F}_{p}$. The proof is now complete.

Proposition. If the minimal polynomial $m$ of a periodic sequence $S=$ $\left\{s_{0}, s_{1}, \cdots\right\}$ is irreducible then the minimal polynomial of $S^{p}=\left\{s_{0}^{p}, s_{1}^{p}, \cdots\right\}$ is $\sigma(m)$, which is also irreducible.

Proof: Since $m$ is irreducible, $\sigma(m)$ is irreducible. On the other hand if

$$
s_{i+n}+c_{n-1} s_{i+n-1}+\cdots+c_{0} s_{i}=0
$$

then

$$
s_{i+n}^{p}+c_{n-1}^{p} s_{i+n-1}^{p}+\cdots+c_{0}^{p} s_{i}^{p}=0 .
$$

Therefore, $\sigma(m)$ is a characteristic polynomial of $S^{p}=\left\{s_{0}^{p}, s_{1}^{p}, \cdots\right\}$. But $\sigma(m)$ is irreducible so it should be the minimal polynomial of $S^{p}=\left\{s_{0}^{p}, s_{1}^{p}, \cdots\right\}$. The proof is now complete.

Theorem 4. If the minimal polynomial $f$ of $S=\left\{s_{0}, s_{1}, \cdots\right\}$ is irreducible then the minimal polynomial of $S+S^{p}+S^{p^{2}}+\cdots+S^{p^{k(f)-1}}$ is $f \sigma(f) \sigma^{2}(f) \cdots \sigma^{k(f)-1}(f)$ which is in $\mathbb{F}_{p}[x]$ and irreducible over the prime field $\mathbb{F}_{p}$. Hence, the comlexity of the sum $S+S^{p}+S^{p^{2}}+\cdots+S^{p^{k(f)-1}}$ is $c(S) k(f)$.

Proof: $\quad$ Since $f$ is irreducible, $\sigma(f), \sigma^{2}(f), \cdots, \sigma^{k(f)-1}(f)$ are all irreducible. But they are the minimal polynomials of $S, S^{p}, S^{p^{2}}, \cdots, S^{p^{k(f)-1}}$, respectively. Hence, $f, \sigma(f), \sigma^{2}(f), \cdots, \sigma^{k(f)-1}(f)$ are pairwise relatively prime and the minimal polynomial of the sum $S+S^{p}+S^{p^{2}}+\cdots+S^{p^{k(f)-1}}$ is $f \sigma(f) \sigma^{2}(f) \cdots \sigma^{k(f)-1}(f)$. The proof is now complete.

Proposition. If the minimal polynomial $f$ of a periodic sequence over $\mathbb{F}_{q}$ is irreducible then $p^{k(f) \operatorname{deg}(f)}-1$ is a period of this sequence.

Proof: Apply Theorem 2 in the special case where the characteristic polynomial of the matrix $A$ is irreducible and replace $q$ by $p^{k(f)}$. This application is possible because $f \in \mathbb{F}_{p^{k(f)}}[x]$ and $f$ is irreducible over there. 
Definition. The order of a polynomial $f \in \mathbb{F}_{q}[x]$ with $f(0) \neq 0$ is the smallest positive integer $\ell$ such that $f$ divides $x^{\ell}-1$. Let ord $(f)$ denote the order of $f$. If $f$ is the minimal polynomial of a periodic sequence over $\mathbb{F}_{q}$ then $\operatorname{ord}(f)$ is the minimal period of this sequence. If $f$ and $g$ are relative prime then $\operatorname{ord}(f g)$ is the least common multiple of ord $(f)$ and ord $(g)$. Moreover, if $x^{\ell}-1$ is divisible by $f$ then it is also divisible by $\sigma(f)$. Therefore, the order of $f$ is divisible by the order of $\sigma(f)$ and so on. Therefore, ord $(f)=\operatorname{ord}(\sigma(f))$.

Theorem 5. The minimal period of a periodic sequence over $\mathbb{F}_{q}$ is $q^{N}-1$ iff its minimal polynomial is a product of distinct irreducible factors such that the degree of each factor divides $N$ and the least common multiple of orders of factors is exactly $q^{N}-1$.

Proof: First we assume that $m=m_{1} m_{2} \cdots m_{h}$ is the minimal polynomial of a periodic sequence such that $m_{1}, m_{2}, \cdots, m_{h}$ are distinct irreducible polynomials of degrees dividing $N$. Then it follows from Theorem 2 that $q^{N}-1$ is a period of this sequence. Moreover, the minimal period of any periodic sequence is the order of its minimal polynomial. Here, the order of $m=m_{1} m_{2} \cdots m_{h}$ is the least common multiple of orders of $m_{1}, m_{2}, \cdots, m_{h}$ which is exactly $q^{N}-1$. Hence, the minimal period is $q^{N}-1$. Now assume that the minimal period of a periodic sequence over $\mathbb{F}_{q}$ is $q^{N}-1$. Then the minimal polynomial of this sequence divides $x^{q^{N}-1}-1$. But $x^{q^{N}}-x$ itself is a square free product of irreducible polynomials of degrees dividing $N$ so the minimal polynomial is a product of distinct irreducible factors and the degree of each factor divides $N$. Moreover, the order of the minimal polynomial is $q^{N}-1$ and equal to the least common multiple of orders of its factors. The proof is now complete.

Remark. Theorem 5 is presented in [9] in the case where $q=2$ without rigour proof.

Definition. An irreducible polynomial $f \in \mathbb{F}_{q}[x]$ is called primitive if ord $(f)=q^{\operatorname{deg}(f)}-1=p^{n \operatorname{deg}(f)}-1$. Any root of a primitive polynomial of degree $N$ generates the cyclic group $\mathbb{F}_{q^{N}}^{*}$ so the number of primitive polynomials of degree $N$ is equal to the number of positive integers $\ell$ which are co-prime to $q$ and $q^{N}-1$. For example, there are only 2 primitive polynomials of degree 3 over $\mathbb{F}_{2}$. They are $x^{3}+x^{2}+1$ and $x^{3}+x+1$. Since ord $(f)=\operatorname{ord}(\sigma(f))$ and $f$ is irreducible if and only if $\sigma(f)$ is irreducible, we have $f$ is primitive if and only if $\sigma(f)$ is primitive. Recall that $k(f)$ denotes the smallest positive 
integer $\ell$ such that $\sigma^{\ell}(f)=f$. We have $k(f) \mid n$ and $f$ is in $\mathbb{F}_{p^{k(f)}}[x]$ so if $f$ is irreducible then $f$ divides $x^{p^{k(f) \operatorname{deg}(f)}-1}-1$. Therefore, if even more $f$ is primitive then it follows that $k(f)=n$. Moreover, any root of a primitive polynomial $f \in \mathbb{F}_{q}[x]$ of degree $N$ is a generator element of the multiplicative group $\mathbb{F}_{q^{N}}^{*}$ so for any index $i \in\left\{1, \cdots, q^{N}-2\right\}$ there is an another index $h \in\left\{1, \cdots, q^{N}-2\right\}$ such that $x^{h}-x^{i}-1$ is divisible by $f$.

Theorem 6. If $f \in \mathbb{F}_{p^{n}}[x]$ is primitive then $g=f \sigma(f) \sigma^{2}(f) \cdots \sigma^{n-1}(f)$ is in $\mathbb{F}_{p}[x]$ and primitive over the prime field $\mathbb{F}_{p}$.

Proof: We have seen that $g \in \mathbb{F}_{p}[x]$ and it is irreducible over there. The order of $f$ is $p^{n \operatorname{deg}(f)}-1=p^{\operatorname{deg}(g)}-1$ which is exactly the order of $g$ over $\mathbb{F}_{p}$ so $g$ is primitive over $\mathbb{F}_{p}$. The proof is now complete.

Definition. If the minimal polynomial of a periodic sequence is primitive of degree $N$ then we call this sequence a max-sequence of order $N$. Clearly, the minimal period of a max-sequence of order $N$ over $\mathbb{F}_{q}$ is $q^{N}-1$. It is well known that if $S=\left\{s_{0}, s_{1}, \cdots\right\}$ is a max-sequence of order $N$ then there is $\beta \in \mathbb{F}_{q^{N}}^{*}$ such that $s_{j}=\operatorname{Tr}\left(\alpha^{j} \beta\right)=\alpha^{j} \beta+\left(\alpha^{j} \beta\right)^{q}+\left(\alpha^{j} \beta\right)^{q^{2}}+\cdots+\left(\alpha^{j} \beta\right)^{q^{N-1}}$ for every $j=0,1,2 \cdots$. Here, $\alpha$ denotes a root of the minimal polynomial of the max-sequence. Indeed, it follows at once from the fact that the $q$ th power of a determinant over the field $\mathbb{F}_{q}$ is giving by the following formula

$$
\left|\begin{array}{cccc}
a_{11} & a_{12} & \cdots & a_{1 N} \\
a_{21} & a_{22} & \cdots & a_{2 N} \\
\vdots & \vdots & \ddots & \vdots \\
a_{N 1} & a_{N 2} & \cdots & a_{N N}
\end{array}\right|^{q}=\left|\begin{array}{cccc}
a_{11}^{q} & a_{12}^{q} & \cdots & a_{1 N}^{q} \\
a_{21}^{q} & a_{22}^{q} & \cdots & a_{2 N}^{q} \\
\vdots & \vdots & \ddots & \vdots \\
a_{N 1}^{q} & a_{N 2}^{q} & \cdots & a_{N N}^{q}
\end{array}\right| .
$$

It is proved in [2] that for a max-sequence of order $N$ and an index $i \in$ $\left\{1, \cdots, q^{N}-2\right\}$ there is an another index $h \in\left\{1, \cdots, q^{N}-2\right\}$ such that $S+\mathbb{E}^{i}(S)=\mathbb{E}^{h}(S)$. In fact, it follows at once from the fact that $s_{j}+$ $s_{j+i}=\operatorname{Tr}\left(\left(1+\alpha^{i}\right) \alpha^{j} \beta\right)=\operatorname{Tr}\left(\alpha^{h} \alpha^{j} \beta\right)=s_{j+h}$, (because $\alpha$ is a generator element of the multiplicative group $\left.\mathbb{F}_{q^{N}}^{*}\right)$. On the other hand, note that in the trace representation $s_{j}=\operatorname{Tr}\left(\alpha^{j} \beta\right)=\alpha^{j} \beta+\left(\alpha^{j} \beta\right)^{q}+\left(\alpha^{j} \beta\right)^{q^{2}}+\cdots+$ $\left(\alpha^{j} \beta\right)^{q^{N-1}}$, we have $\beta \in \mathbb{F}_{q^{N}}^{*}$ so $\beta$ should be a power of $\alpha$ and consequently, $s_{j}=\operatorname{Tr}\left(\alpha^{j+i_{0}}\right)$ for every $j=0,1,2 \cdots$. Therefore, max-sequences of the same minimal polynomial are equivalent. The number of primitive 
polynomials of degree $N$ will gives us the number of non-equivalent maxsequences of order $N$. This number is equal to the number of positive integers $\ell$ which are co-prime to $q$ and $q^{N}-1$. For example, there are only 2 primitive polynomials of degree 3 over $\mathbb{F}_{2}$. They are $x^{3}+x^{2}+1$ and $x^{3}+x+1$. Therefore, there are only two max-sequences of order 3 over $\mathbb{F}_{2}$, which are 1110100 and 1110010. Finally, we note that the trace operator Tr is a linear functional, which maps $\mathbb{F}_{q^{N}}$ as a vector space (over $\mathbb{F}_{q}$ ) onto $\mathbb{F}_{q}$.

\section{DE BRUIJN SEQUENCES AND THEIR MODIFI- CATION}

A de Bruijn sequence of order $N$ th over $\mathbb{F}_{q}$ is a $q^{N}$-periodic sequence $B=$ $\left\{b_{0}, b_{1}, \cdots\right\}$ such that

$$
\left\{\left(b_{i}, b_{i+1}, \cdots, b_{i+N-1}\right): i=0,1, \cdots, q^{N}-1\right\}=\mathbb{F}_{q}^{N} .
$$

Clearly, the minimal polynomial of any de Bruijn sequence is a power of $x-1$. Moreover, the weight of a de Bruijn sequence $B$ is zero so its complexity $c(B)$ should satisfy $p^{n N-1}<c(B)<p^{n N}$ where $p^{n}=q$. Let

$$
B(x)=b_{0}+b_{1} x+\cdots+b_{q^{N}-1} x^{q^{N}-1} .
$$

Then the minimal polynomial of $B$ is

$$
\frac{x^{q^{N}}-1}{\operatorname{gcd}\left(x^{q^{N}}-1, B(x)\right)}=(x-1)^{c(B)}
$$

so

$$
B(x)=(x-1)^{q^{N}-c(B)} f(x)
$$

where $f$ is co-prime with $x-1$ and $\operatorname{deg}(f)=c(B)-1$. We are interested in a modification of de Bruijn sequences. It is obtained from a de Bruijn sequence by removing a zero from the unique allzero $N$-tuple. The minimal period of a modified sequence of order $N$ is $q^{N}-1$. Hence, by Theorem 5 the minimal polynomial of a modified sequence is a product of distinct irreducible factors such that the degree of each factor divides $N$ and the 
least common multiple of orders of factors is exactly $q^{N}-1$. On the other hand, the minimal polynomial of the modified sequence is

$$
\frac{x^{q^{N}-1}-1}{\operatorname{gcd}\left(x^{q^{N}-1}-1, B(x)\right)}=\frac{x^{q^{N}-1}-1}{(x-1) \operatorname{gcd}\left(x^{q^{N}-1}-1, f(x)\right)}=: m(x) .
$$

It is interesting to study in more details the irreducible factors of the minimal polynomial of a modified de Bruijn sequence.

Theorem 7. Any max-sequence is a modified de Bruijn sequence of the same order. Therefore, there exists a de Bruijn sequence of any order. The number of non-equivalent de Bruijn sequences of order $N$ is at least the number of primitive polynomials of the same order. Recall that a periodic sequence is called a max-sequence if its minimal polynomial is primitive.

Proof: Let $s_{j}=\operatorname{Tr}\left(\alpha^{j+i_{0}}\right)$ be a max-sequence of order $N$. Let $f$ be the minimal polynomial of the max-sequence. Assume that $\operatorname{Tr}\left(\alpha^{\ell-1}\right) \neq 0$ and $\operatorname{Tr}\left(\alpha^{\ell}\right)=\operatorname{Tr}\left(\alpha^{\ell+1}\right)=\cdots=\operatorname{Tr}\left(\alpha^{\ell+N-1}\right)=0$. Then $\operatorname{Tr}\left(\alpha^{\ell+N}\right)=$ $-f(0) \operatorname{Tr}\left(\alpha^{\ell-1}\right) \neq 0$ so the max-sequence is really a modified de Bruijn sequence of the order $N$.

Now we define $B_{N}$ the $q$-ary de Bruijn directed graph of order $N$ as follows. Let the vertices of $B_{N}$ be the elements of $\mathbb{F}_{q}^{N}$. Moreover, a vertex $\left(a_{1}, a_{2}, \cdots, a_{N}\right)$ is directed to another vertex $\left(b_{1}, b_{2}, \cdots, b_{N}\right)$ if $\left(a_{2}, \cdots, a_{N}\right)=$ $\left(b_{1}, b_{2}, \cdots, b_{N-1}\right)$. A de Bruijn sequence of order $N$ is the directed Hamilton cycle in this graph, which is certainly exists! Moreover, the diameter of this graph is $N$. Two non-equivalent de Bruijn sequences will give two distinct Hamilton cycles. For example, there are only two non-equivalent de Bruijn sequences of order 3 over $\mathbb{F}_{2}$ are 11101000 and 11100010 . Hence, the number of Hamilton cycles in $B_{N}$ is at least the number of primitive polynomials of order $N$. The number of directed Hamilton cycles in $B_{N}$ is at least the number of positive integers $\ell$ which are co-prime to $q$ and $q^{N}-1$. Now we define the difference operator

$$
\mathbb{D}: \mathbb{F}_{q}^{N} \rightarrow \mathbb{F}_{q}^{N-1}
$$

by setting $\mathbb{D}\left(a_{1}, \cdots, a_{N}\right)=\left(a_{2}-a_{1}, \cdots, a_{N}-a_{N-1}\right)$. Then $\mathbb{D}$ induces an graph homomorphism from $B_{N}$ onto $B_{N-1}$. Therefore, any de Bruijn sequence of order $N$ induces a de Bruijn sequence of order $N-1$. For example, two 
sequences 11101000 and 11100010 of order 3 induce the same sequence 0011 of order 2 .

\section{CONVOLUTION OF PERIODIC SEQUENCES}

For two sequences $S=\left\{s_{0}, s_{1}, \cdots\right\}$ and $T=\left\{t_{0}, t_{1}, \cdots\right\}$ we define their convolution $S * T$ to be the sequence $U=\left\{u_{0}, u_{1}, \cdots\right\}$ by letting

$$
u_{\ell}=s_{\ell} t_{0}+s_{\ell-1} t_{1}+\cdots+s_{0} t_{\ell} \quad \text { for } \ell=0,1,2, \cdots .
$$

For example, the convolution of $\left\{1,3,3^{2}, \cdots\right\}$ with itself over $\mathbb{F}_{5}$ is the Fibonacci sequence $\{1,1,2,3, \cdots\}$. Recall that $\mathcal{M}(f)$ denotes the vector space of periodic sequences having $f$ as a characteristic polynomial. Here $f \in \mathbb{F}_{q}[x]$ is a monic polynomial. The dimension of $\mathcal{M}(f)$ over $\mathbb{F}_{q}$ is $\operatorname{deg}(f)$. It is proved in [6] that

$$
\mathcal{M}(f) * \mathcal{M}(g) \subseteq \mathcal{M}(f g) \quad \text { for all monic polynomials } f, g \in \mathbb{F}_{q}[x] .
$$

This result is the best. For example, over $\mathbb{F}_{5}$ we have

$$
\mathcal{M}(x-3) * \mathcal{M}(x-3) \neq \mathcal{M}\left((x-3)^{2}\right) .
$$

Moreover, it is proved in [7] that

$$
\mathcal{M}(x f(x)) * \mathcal{M}(g)=\mathcal{M}(f g) \quad \text { for all monic polynomials } f, g \in \mathbb{F}_{q}[x] .
$$

A typical example of convolution is Catalan number

$$
c_{n}=\frac{1}{n+1}\left(\begin{array}{c}
2 n \\
n
\end{array}\right) \text {. }
$$

It is well known that

$$
c_{\ell+1}=c_{\ell} c_{0}+c_{\ell-1} c_{1}+\cdots+c_{0} c_{\ell} \quad \text { for } \ell=0,1,2, \cdots
$$

so in our notation $\mathbb{E}(C)=C * C$ where $C=\left\{c_{0}, c_{1}, \cdots\right\}$.

Acknowledgement. Deepest appreciation is extended towards the NAFOSTED (the National Foundation for Science and Techology Development in Vietnam) for the financial support. 


\section{References}

[1] L. Berg and S. Stevic, Linear diiference equations mod 2 with applications to nonlinear difference equations, J. Difference Equ. Appl. $\mathbf{1 4}(2008)$ 693-704.

[2] S. R. Blackburn A Note on Sequences with the Shift and Add Property 9 (1996) 251-256.

[3] S. R. Blackburn, T. Etzion and K. G. Paterson, Permutation Polynomials, de Bruijn Sequences, and Linear Complexity, Journal of Combinatorial Theory, Series A, 76 (1996) 55-82.

[4] Fang-Wei Fu, Harald Niederreiter and Ming Su, The expectation and variance of the joint linear complexity of random periodic multisequences Journal of Complexity, Volume 21, Issue 6, December 2005, Pages 804822 .

[5] R. Goodman et al, Representations and invariants of the classical groups, Cambridge University Press, 2001.

[6] P. Haukkanen, On a convolution of linear recurring sequences over finite fields, Journal of Algebra 149 (1992) 179-182.

[7] P. Haukkanen, On a convolution of linear recurring sequences over finite fields, II Journal of Algebra 164 (1994) 542-544.

[8] V. Kocic and G. Ladas, Global behavior of nonlinear difference equations of higher order with applications. Mathematics and its Applications, 256. Kluwer Academic Publishers Group, Dordrecht, 1993.

[9] G. M. Kyureghyan, Minimal polynomials of the modified de Bruijn sequences, Discrete Applied Mathematics, 156 (2008) 1549-1553.

[10] R. Lidl and H. Niederreiter, Finite fields. With a foreword by P. M. Cohn. Second edition. Encyclopedia of Mathematics and its Applications 20. Cambridge University Press, Cambridge, 1997.

[11] H. Niederreiter, Random number generation and quasi-Monte Carlo methods, CBMS-NSF Regional Conference Series in Applied Mathematics, 63. Society for Industrial and Applied Mathematics (SIAM), Philadelphia, PA, 1992. vi+241 pp. ISBN: 0-89871-295-5. 
[12] K. G. Paterson, Perfect factors in de Bruijn graph, Design, Codes and Cryptogr. 5 (1995) 115-138.

[13] A.M. Robert, A course in $p$-adic analysis, Springer 2000. 